\title{
Effect of the Pauli exclusion principle on the singlet exciton yield in conjugated polymers
}

\author{
A. Thilagam* \\ Future Industries Institute, \\ University of South Australia, \\ Adelaide 5095, South Australia
}

\begin{abstract}
Optical devices fabricated using conjugated polymer systems give rise to singlet exciton yields which are high compared to the statistically predicted estimate of $25 \%$ obtained using simple recombination schemes. In this study we evaluate the singlet exciton yield in conjugated polymers systems by fitting to a model that incorporates the Pauli exclusion principle. The rate equations which describe the exciton dynamics include quantum dynamical components (both density and spin-dependent) which arise during the spin-allowed conversion of composite intra-molecular excitons into loosely bound charge-transfer (CT) electron-hole pairs. Accordingly, a crucial mechanism by which singlet excitons are increased at the expense of triplet excitons is incorporated in this work. Non-ideal triplet excitons which form at high densities, are rerouted via the Pauli exclusion mechanism to form loosely bound CT states which subsequently convert to singlet excitons. Our derived expression for the yield in singlet exciton incorporates the purity measure, and provides a realistic description of the carrier dynamics at high exciton densities.
\end{abstract}

PACS numbers: 78.67.-n, 77.22.-d

\section{INTRODUCTION}

The properties of singlet and triplet excitons (correlated electron-hole quasiparticles) in organic systems ${ }^{1-\underline{\underline{8}}}$ play primary roles in determining the electroluminescent quantum efficiencies of organic optical devices fabricated using $\pi$-conjugated polymers $\frac{6.7 .9-14}{14}$. Two fermions of spin $\frac{1}{2}$, give rise to four microstates: one singlet state (antisymmetric under particle exchange) and three triplet states (symmetric under particle exchange). Unlike the triplet exciton, the singlet exciton undergoes rapid radiative recombination producing light. Accordingly, the ratio of singlet to triplet exciton formation rates, $\gamma$, provides a useful quantitative measure of the electroluminescence efficiency in conjugated polymers $\frac{15,16}{}$. Based on the assumption that the formation cross sections of singlet and triplet excitons are the same, $\frac{1}{4}$ of available bi-fermion pairs (or charge carriers) will form singlet excitons with the well documented statistical yield of $\frac{1}{1+3}(25 \%)$. However several works $\frac{17,18}{}$ have shown evidence of internal quantum efficiencies of $25 \%$ or more. To this end, the exact processes of spin dynamics that determines singlet exciton yield and the electroluminescence efficiencies are not fully resolved.

The conversion dynamics between the singlet and triplet excitonic states, related intermediate states which present as loosely bound electron-hole pairs, as well as the recombination lifetimes of all known states are fundamental to evaluating the yields of singlet exciton states. Several studies ${ }^{3,8,19,20}$ have examined the underlying mechanisms for the high singlet exciton yields $(>25 \%)$ observed in experimental results involving conjugated polymer systems. The decrease of a spin-dependent $\gamma$ was associated with strong spin-orbital coupling effects in an earlier work ${ }^{20}$. By taking into account the optical interference effects on radiative rates, a higher singlet exciton yield (35\%-45\%) was obtained in conjugated polymers $\underline{19}$. The singlet exciton generation is lower in monomers compared to its generation in polymers. This may be linked to the fact that recombination dynamics is spin-independent in monomers, while it becomes spin-dependent in polymers ${ }^{2}$. These differences highlight the dependence of exchange interactions and other electronic properties on the molecular configuration of the polymer chains ${ }^{21}$. The energy relaxation process involving the singlet exciton is rapid unlike the slower relaxation via the low lying triplet exciton ${ }^{22}$. This factor may contribute partly to differences in dynamics of the two spin states of the exciton. It appears that several underlying processes account for the variations in singlet exciton yield, which also dependes on the material system under investigation. Thus far the inclusion of the Pauli-exclusion principle which becomes dominant at higher exciton densities has not been incorporated in all earlier works $\frac{3,8,19,20}{}$. The importance of the Pauli-exclusion principle at increasing exciton densities and its neglect in earlier works forms the key motivation for the current study.

While two or more ideal excitons can occupy the same quantum state, the multiple occupation of single-particle states is forbidden for several fermions by virtue of the Pauli-blocking mechanism. The rapid absorption of photons under intense illumination conditions excites multiple pairs of electrons and holes, generating closely spaced excitons. The decrease in distance between the optically generated electron-pair pairs. results in non-ideal bosonic excitons that acquire fermionic attributes. The ideal boson commutation relations which are applicable to the structureless exciton need to be modified to non-ideal commutation forms due to the operation of the Pauli exclusion principle ${ }^{23}$. 
While the exclusion principle operates under all conditions, it does not influence the dynamics of almost ideal excitons at very low densities.

The quantum statistical attributes of the coboson such as the composite exciton ${ }^{24}-26$ has received increased interest in recent works $33,27-32$. These studies examined the links between entanglement and composite nature of bosons with non-ideal attributes. A key result is that quantum entanglement underpins the binding of constituent fermions to form the composite boson ${ }^{23,27}$. While large entanglement between constituent fermions gives rise to a point-like structureless ideal boson, the less entangled electron-hole pair represents the non-ideal composite boson. Quantum entanglement of the fermions constituents is thus critical to ensuring the bosonic attribute of the composite system remains intact 23,33 .

In this work, the concept of entanglement is incorporated via the purity measure $0 \leq P \leq 1$ to examine quantum processes in organic materials. The purity measure is linked to the number of Schmidt modes ${ }^{23,27}$ associated with the entanglement of the paired structure of the exciton or any other species of composite boson. An exciton with zero or very small purity $\rightarrow 0$ behaves as an ideal boson, while non-zero values of $P$ describe a non-ideal composite boson. A loosely bound electron-hole pair is associated with the less entangled electron and hole system with $P>0$. In a system of many excitons, the purity $P$ quantifies the correlations between the fermions, with $P \approx 0$ implicating a highly correlated system of ideal excitons. For $N$ excitons that occupy the same quantum state, the exciton can be considered a boson as long as $N P \ll 1^{23}$. Accordingly, $\frac{1}{P}$ can be interpreted as the number of particles that can occupy the same pure state, before the Pauli blocking mechanism begins to influence the idealized nature of the bosons. Multiple singlet and triplet excitons generated in organic molecules experience a crossover to non-ideal bosonic states as the excitons are confined to spaces which are comparable to their size $e^{34.35}$. In this study, we employ a composite boson model of the exciton to examine the singlet exciton yield at non-zero values of the purity $P$. The incorporation of entanglement aspects in this study will help overcome the shortcomings of conventional models used to model correlated system behavior in solids.

We consider the model of inter-chain electron-hole interaction between pairs of parallel polymers, where "chargetransfer" (CT) exciton states exist at intermediate energies levels positioned between the electron-hole continuum and the strongly bound exciton states ${ }^{8}$. The singlet and triplet charge-transfer (CT) quasi-particles appear as critical precursors to the tightly bound exciton states in polymers. We extend this model by incorporating a mechanism by which tightly bound excitons convert to the more loosely bound CT intermediate states with loss of pure bosonic attributes. The conversion occurs due to operation of the Pauli exclusion principle, with the charge-transfer states possessing a higher degree of fermionic attributes.

The energetic difference between singlet and triplet exciton which arise from the opposite sign of the exchange interaction is generally high $(\approx 0.7 \mathrm{eV})$ in conjugated polymers 36 . The "charge-transfer" $(\mathrm{CT})$ singlet and triplet states, on the other hand are formed at approximately the same energy levels ${ }^{8}$ due to the weak exchange interactions of these states. Inter-conversion between the two types (singlet, triplet) of intermediate CT states may occur soon after their formation. Typical exciton lifetimes for organic materials such as poly(p-phenylene vinylene), PPV $\underline{37}$ and anthracene ${ }^{38}$ are of the order of $1 \mathrm{~ns}^{39}$. In other organic materials such as PDHFV, PTEH and PDHFHPPV $\underline{40}$, the exciton lifetimes can range from 0.05 to $0.71 \mathrm{~ns}$.

In this work, we consider that the Pauli exclusion operational times may proceed faster than the exciton lifetimes at high densities. The Pauli exclusion related conversion of one excitonic species to another becomes favorable over excitonic interactions with phonons or impurities with increased deviations in the bosonic attributes of excitons. Depending on the purity, $P$ of the excitons and the material system ${ }^{39}, 40$ under study, Pauli exclusion based conversions are expected to proceed at times in the order of 0.01 to $1 \mathrm{~ns}$. These processes are expectedly absent at low densities due to the ideal bosonic nature of the excitons. The results in this study are thus relevant to excitons which become non-ideal bosons as the excitonic purity is increased. In this study, we provide results of the singlet exciton yield for range of conversion times linked to the Pauli mechanism. The main results of this work is the derivation of an expression for the singlet exciton yield which incorporates the purity measure.

\section{RATE EQUATIONS INCORPORATING THE PAULI EXCLUSION PRINCIPLE}

In typical organic devices, the injection of charges carriers precedes the formation of excitons which diffuse for an amount of time before decaying radiatively (singlet exciton) or non-radiatively (triplet exciton). The overall external quantum efficiency of the organic devices is written as a product, $\eta_{f} \eta_{s} \eta_{p} \eta_{o}$ where $\eta_{f}$ is the efficiency of exciton formation from free carriers, $\eta_{s}$ is the singlet exciton yield, $\eta_{p}$ is the photoluminescence quantum efficiency and $\eta_{o}$ is the fraction of of photons which are emitted without being utilized by the device. When the exciton density becomes high enough, the manifestation of the Pauli exclusion principle can be examined through $\eta_{f}$ and $\eta_{o}$, with incorporation of relevant device parameters. In this work, we focus on the effects of the Pauli exclusion principle on the underlying processes which determine $\eta_{s}$. To evaluate $\eta_{s}$, we consider a typical setup in which both singlet and triplet excitons 


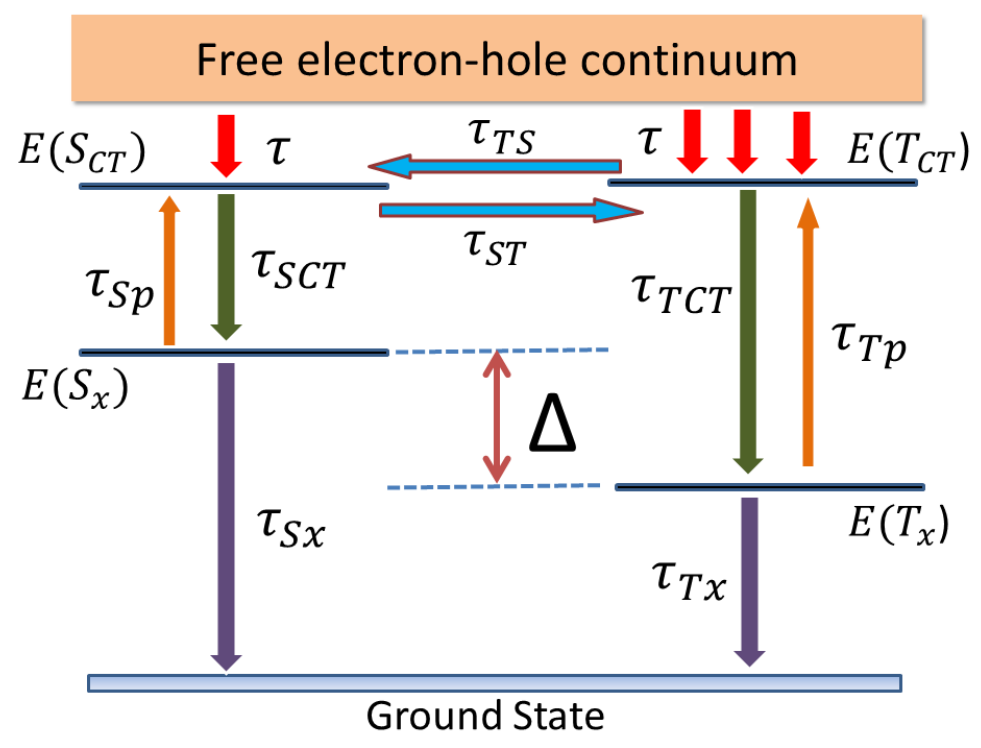

FIG. 1: Schematics of the energy levels $(E(i))$ of singlet $\left(S_{X}\right)$ and triple $\left(T_{X}\right)$ exciton states and singlet $\left(S_{C T}\right)$ and triplet $\left(T_{C T}\right)$ "charge-transfer" states. The conversion times within the singlet states $\left(\tau_{S C T}\right)$ and triplet states $\left(\tau_{T C T}\right)$ are indicated by the green arrows. The unequal times of inter-conversions between the spin CT states $\left(\tau_{T S}\right.$, $\left.\tau_{S T}\right)$ are indicated by the blue arrows. The Pauli exclusion reversal times $\left(\tau_{T p}\right.$ and $\left.\tau_{S p}\right)$, which are present only at non-zero exciton purity $p$, are denoted by the orange arrows, while the recombination times of the singlet and triplet excitons $\left(\tau_{S x}, \tau_{T x}\right)$ are shown via the purple arrows. $\Delta$ denotes the exchange energy between $S_{X}$ and $T_{X}$.

are involved in a coordinated process to produce the optimal singlet exciton yield.

The schematics of the energy levels of singlet and triple exciton states and "charge-transfer" (CT) states for the model under study is shown in Fig 1 . We employ several notations adopted in Ref. $\stackrel{8}{-}$ for exciton and CT states, and assume that charge carriers which are injected into the polymer material possess random spin orientations. The injected charge carriers form the loosely bound charge-transfer singlet and triplet quasi-particles, $S_{C T}$ and $T_{C T}$. The inter-system-crossing between $T_{C T}$ and $S_{C T}$ due to the spin-orbit coupling mechanism is considered unequal, where $\tau_{T S}\left(\tau_{S T}\right)$ denotes the time taken for $T_{C T}\left(S_{C T}\right)$ state to convert to the $S_{C T}\left(T_{C T}\right)$ state. The conversion from $T_{C T}$ to the triplet exciton, $T_{X}$ occurs at the rate, $1 / \tau_{T C T}$, while the conversion from $S_{C T}$ to the triplet exciton, $S_{X}$ occurs at the rate, $1 / \tau_{S C T}$. The recombination of the singlet (triplet) exciton state $S_{X}\left(T_{X}\right)$ at energy level, $E\left(S_{X}\right)\left(E\left(T_{X}\right)\right)$ to the ground state occurs in the time, $\tau_{S x}\left(\tau_{T x}\right)$.

We consider the occurrence of Pauli exclusion reversal times $\tau_{T p}$ and $\tau_{S p}$ for the triplet and singlet exciton states respectively at non-zero exciton purity $\left(p_{t}, p_{s}\right)$. Depending on the degree of exciton purity or compositeness, the reversal times may proceed faster than the times of $T_{r}, \tau_{S x}, \tau_{T x}$. The reversal times at the non-zero exciton purity $\tau_{T p}$ and $\tau_{S p}$ are indicated by the upward arrows in Fig 1. Both these reversal times do not apply at low exciton densities, for which the recombination processes, $S_{C T} \rightarrow S_{X}$ and $T_{C T} \rightarrow T_{X}$ occur uninterrupted due to the almost ideal bosonic exciton. At high enough exciton densities where the purity assume non-trivial values, Pauli exclusion related processes become favorable compared to exciton decoherence due to interaction with phonons and/or impurities. The role of the times, $\tau_{T S}, \tau_{S T}, \tau_{S C T}, \tau_{T C T}, \tau_{T p}$ and $\tau_{S p}$ in enhancing the singlet exciton yield will be examined assuming the absence of coherence between the singlet and triplet CT states.

We first consider the simplified model with equal times of inter-conversions between the spin CT states, so that $\tau_{T S}=\tau_{S T}=\tau_{I S C}$. The set of coupled equations relating the number of various quasiparticle states such as singlet 
excitons $\left(N_{S X}\right)$, triplet excitons $\left(N_{T X}\right)$, singlet CT states $\left(N_{S C T}\right)$ and triplet CT state $\left(N_{T C T}\right)$ appear as

$$
\begin{aligned}
\frac{d N_{S C T}}{d t} & =\frac{N_{i}}{4 \tau}+\frac{N_{T C T}}{\tau_{I S C}}-N_{S C T}\left(\frac{1}{\tau_{I S C}}+\frac{1}{\tau_{S C T}}\right)+\frac{P_{s} N_{S X}}{\tau_{S p}} \\
\frac{d N_{T C T}}{d t} & =\frac{N_{i}}{2 \tau}+\frac{N_{S C T}}{\tau_{I S C}}-N_{T C T}\left(\frac{1}{\tau_{I S C}}+\frac{1}{\tau_{T C T}}\right)+\frac{P_{t} N_{T X}}{\tau_{T p}} \\
\frac{d N_{S X}}{d t} & =\frac{N_{S C T}}{\tau_{S C T}}-\frac{N_{S X}}{\tau_{S X}}-\frac{P_{s} N_{S X}}{\tau_{S p}} \\
\frac{d N_{T X}}{d t} & =\frac{N_{T C T}}{\tau_{T C T}}-\frac{N_{T X}}{\tau_{T X}}-\frac{P_{t} N_{T X}}{\tau_{T p}}
\end{aligned}
$$

where $\frac{N_{i}}{\tau}$ is the initial number electron-hole pairs created per second. To reduce the number of coupled equations, we have used a single term $\left(N_{T C T}\right)$ to denote the two possible triplet terms. In Eqs. 1 to 4 , we have considered an exciton number dependent term which appears as

$$
\frac{p_{i} N_{j X}}{\tau_{j p}},
$$

where $N_{j X}$ denotes the number of singlet exciton $\left(N_{S X}\right)$ or triplet excitons $\left(N_{T X}\right)$. The purity measure $P_{s}\left(P_{t}\right)$ is associated with the singlet (triplet) non-ideal exciton. In order to obtain quantitative results in forthcoming sections, we consider that the Pauli exclusion reversal times $\tau_{T p}$ and $\tau_{S p}$ to be of the same order as the recombination times of the singlet and triplet excitons $\tau_{S x}, \tau_{T x}$. The form of Eq. 5 is based on the lower bound of the normalization ratios associated with $(N+1)$ and $N$ exciton which will be discussed next.

\section{A. Pauli exclusion purity factors, $P_{s}$ and $P_{t}$}

The purity measures, $P_{s}$ and $P_{t}$ in Eqs. 1, 4 of the non-ideal singlet and triplet excitons created from the "chargetransfer" states appear in the derived relation 27

$$
1-P_{i} \cdot N \leq \frac{\chi_{N+1}}{\chi_{N}} \leq 1-P_{i}
$$

where $i=\mathrm{s}$ (singlet) or t (triplet), and $\chi_{N}$ is the normalization term linked to the superposed state of $N$ non-ideal excitons ${ }^{23,27}$. The deviations from unity in the ratio, $\frac{\chi_{N+1}}{\chi_{N}}$ yield a measure of the non-bosonic quality of the correlated electron-hole pair ${ }^{23,24,27}$, establishes a link with with entanglement. The upper and lower bounds to $\frac{\chi_{N+1}}{\chi_{N}}$ in Eq. 6 appear in terms of the purity $P_{i}$ of the single-fermion reduced state. The ratio $\frac{\chi_{N+1}}{\chi_{N}}$ can be interpreted as the effective probability of increasing the number of excitons from $N$ to $N+1$ in a system. Excitons with ideal boson properties are described by an $N$ independent term $\chi_{N}=1$ so that $P_{s}=P_{t}=0$. The lower bound in Eq. 6 decreases monotonically with $N$, and vanishes at $P=\frac{1}{N}$.

The coherence between the $N$ excitons is decreased when the $(N+1)$ st exciton is added. This is due to the Pauli exclusion principle operating with the probability, $1-\frac{\chi_{N+1}}{\chi_{N}}$. The importance of incorporating density dependent attributes, $P_{i}$ (i=s,t) in analyzing the singlet exciton yield in Eqs. 1 to 4 is highlighted by Eq. 6. The density dependent attributes, $P_{i}(\mathrm{i}=\mathrm{s}, \mathrm{t})$ therefore has to be taken into account in any analysis of the singlet or triplet exciton yield under strong illumination conditions.

At high purities, the bounds in Eq. 6 becomes less efficient $30,31,41$ as the upper and lower bound terms differ significantly from each other. The purity $P$ alone is not sufficient to provide a reliable measure of the bounds in Eq. 6. In this regard $P$ cannot be used to accurately predict the bosonic attribute of excitons. To further exploit the simplicity of Eq. 6. we restrict our study to excitonic systems of small purity. For the purpose of illustrating the explicit role of the excitonic purity term, we consider an $N$ independent upper bound for the normalization ratio

$$
\frac{\chi_{N+1}}{\chi_{N}} \approx 1-P_{i}
$$

which is applicable to a system of interacting excitons with minimal deviations from ideal bosonic attributes. This simple model is used to derive an analytical form for the exciton yield. Subsequently, we utilize a realistic numerical model which is applicable at small purity based on the relation

$$
P \approx \frac{\zeta}{N}
$$

where $0<\zeta<1$. We next employ Eqs. 1, 4 as well as Eqs. 7 and 8 to obtain quantitative results of the exciton yield. 


\section{B. Analytical form of the singlet exciton yield using Eq. 7}

Using Eq 7 which is applicable for system of weakly interacting bosons, and solving Eqs. 1 to 4 under the steady state conditions we obtain the singlet exciton yield $\eta_{s}$ as

$$
\begin{aligned}
\eta_{s} & =\frac{N_{S X} / \tau_{S X}}{N_{i} / \tau} \\
& =\frac{\gamma+3 P_{t} \alpha_{t}+3}{4\left(\beta+\gamma-\beta P_{s} \alpha_{s}-2 \gamma P_{s} \alpha_{s}-2 P_{s} \alpha_{s}-2 P_{s} P_{t} \alpha_{s} \alpha_{t}+P_{t} \alpha_{t}+1\right)},
\end{aligned}
$$

where $\beta=\frac{\tau_{S C T}}{\tau_{T C T}}$ and $\gamma=\frac{\tau_{I S C}}{\tau_{T C T}}, \alpha_{s}=\frac{\tau_{S X}}{\tau_{S p}}$ and $\alpha_{p}=\frac{\tau_{T X}}{\tau_{T p}}$. At $P_{s}=P_{t}=0$, Eq. 9 reduces to the form

$$
\eta_{s}=\frac{3+\gamma}{4(1+\beta+\gamma)}
$$

which was earlier derived in Ref료. In Fig. 2ha, the singlet yield is obtained as a function of $P_{s}=P_{t}=P_{i}$ at $\beta=$ 0 ( or $\tau_{T C T} \gg \tau_{S C T}$ ), and for equivalent Pauli reversal times to recombination times, $\alpha_{s}=\alpha_{p}=1$. Fig. $2 \mathrm{~b}$ shows the singlet yield as a function of $\gamma=\frac{\tau_{I S C}}{\tau_{T C T}}$ at two different values of $P_{s}=P_{t}=P_{i}$. We restrict the purity values to the range $0 \leq P \leq 0.15$ in Figs. $2 \mathrm{a}, \mathrm{b}$ as Eq. 9 holds valid only for small values of $P$. The results in Figs. 2a, $\mathrm{b}$ highlight the importance of taking into account the Pauli exclusion mechanism for non-ideal excitons. A gradual increase in the singlet exciton yield occurs even with small increase in the purity values, $P_{s}$ and $P_{t}$. This increase in the singlet exciton yield is further enhanced at lower $\gamma$. These preliminary results suggest that the spin-allowed route $T_{X} \rightarrow T_{C T} \rightarrow S_{C T} \rightarrow S_{X}$ facilitates the increase in $\eta_{s}$. These findings can be compared with those of earlier works $8,19,20$ where an increase in the singlet exciton yield is attributed to transfer of excitation from an intermediate triplet state rather than from the tightly bound triplet state.

The model used to derive results for Fig. 2 $2 \mathrm{a}, \mathrm{b}$ is only applicable for small deviations in bosonic attributes of the excitons. While we expect saturation in the singlet exciton yield to occur at higher $P$ resulting in $\eta_{s} \rightarrow 1$, this cannot be predicted within the simple model used here. The usefulness of Eq. 9 is highlighted by the fact that it quantifies the mechanism by which there is increased proportion of singlet excitons through decrease in triplet exciton recombination. The triplet excitons are instead rerouted as a result of the Pauli exclusion principle to form loosely bound CT states which subsequently convert to singlet excitons. As a consequence, the fluorescence quantum yield can reach estimates of even $50 \%$ or more, which is the main result of this work.

\section{Numerical solution for population of exciton species using Eq. 8}

Using numerical techniques, we determine the population of all species, $N_{k}(k=S X, T X, T C T, S C T)$ using Eqs. 1 to 4 and substituting $P_{s}$ and $P_{t}$ with the $N$-dependent form in Eq 8 We use typical parameters estimates for organic materials such as poly(p-phenylene vinylene), $\mathrm{PPV} \underline{37}$ and anthracene $\underline{38}$ where the exciton lifetime is of the order of 1 $\mathrm{ns}^{39}, \tau_{T C T} \approx 10 \mathrm{~ns}^{-}, \tau_{S C T} \approx 1-10 \mathrm{ps}^{38}$ and $\tau_{I S C} \approx 0.1-10 \mathrm{~ns}^{36,38}$.

The evolution of $N_{k}(k=S X, T X, T C T, S C T)$ with time, $t$ is shown in Fig. 3. While we set $\zeta=0$ in Fig. $3 \mathrm{k}$, a non-zero $\zeta=0.15$ (which reflects higher exciton purity) is used to obtain the results shown in Fig. 3b. All other parameters are provided in the caption of Fig. 3, in particular, we chose $\tau_{T C T}=10 \mathrm{~ns}$ and $\tau=10 \mathrm{~ns}$. Accordingly the time of evolution, $t$ appears in units of $10 \mathrm{~ns}$ and the population of all species, $N_{k}$ are taken relative to the initial number electron-hole pairs, $N_{i}=1$. We note that for the chosen set of parameters, the singlet exciton population rises rapidly and reaches a steady state at $t \approx 30 \mathrm{~ns}$. The triplet exciton which shows appreciable loss even at the beginning stage of evolution, is not apparent in Fig. 3a,b. The population of the triplet CT species appears higher than the singlet CT species, for the set of parameters employed to solve Eqs. 1 to 4. A saturation of the single exciton population occurs beyond large times $t>35$ ns for non-zero purity $P_{s}=P_{t}$.

In contrast to Fig. $3 \mathrm{k}$, b where we used $\beta=\frac{\tau_{S C T}}{\tau_{T C T}}=0.001$, the results in Fig. $4 \mathrm{a}, \mathrm{b}$ are obtained using an increased

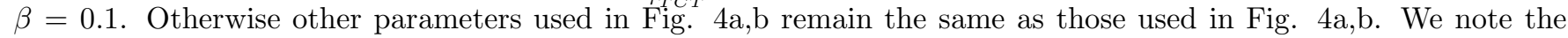
prominent appearance of the triplet exciton with increase in $\beta$ in Fig. 柏. Furthermore, with increase in the exciton purity (non-zero $\zeta$ ), the population of the triplet exciton exceeds that of the singlet CT species. The results obtained here indicates the possibility of converting incoming photons to singlet excitons with minimal energy dissipation with onset of the Pauli exclusion mechanism under suitable conditions. The results obtained in this study clearly demonstrates the critical role of triplet excitons when present at high densities. We make clear that all results obtained in this study are valid for small exciton purity estimates. The model employed in this study therefore stands valid for non-ideal excitons which depart slightly from possessing ideal boson behavior. 


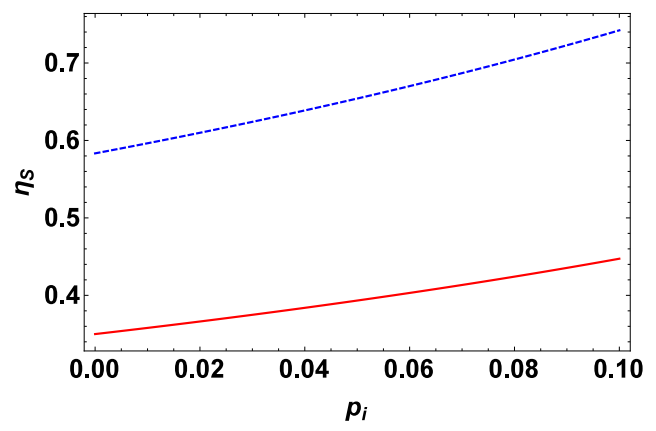

(a)

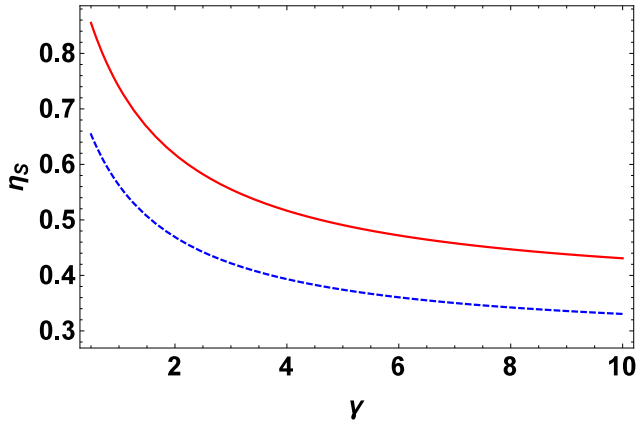

(b)

FIG. 2: (a) The singlet exciton yield, $\eta_{s}$ as a function of $P_{s}=P_{t}=P_{i}$ obtained using Eq9 The red solid line corresponds to $\gamma=\frac{\tau_{I S C}}{\tau_{T C T}}=4$, while the blue dashed line corresponds to $\gamma=0.5$. (b) The singlet exciton yield, $\eta_{s}$ as a function of $\gamma=\frac{\tau_{I S C}}{\tau_{T C T}}(\mathrm{Eq} 9)$. The red solid line corresponds to $P_{i}=0.15$, while the blue dashed line corresponds to $P_{i}=0.05$. For both figures, we set $\alpha_{s}=\alpha_{p}=1\left(\alpha_{s}=\frac{\tau_{S X}}{\tau_{S p}}, \alpha_{p}=\frac{\tau_{T X}}{\tau_{T p}}\right)$ and $\beta=0$.

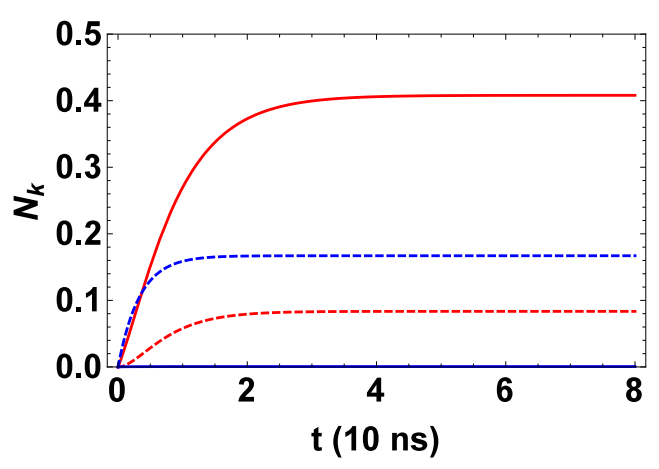

(a)

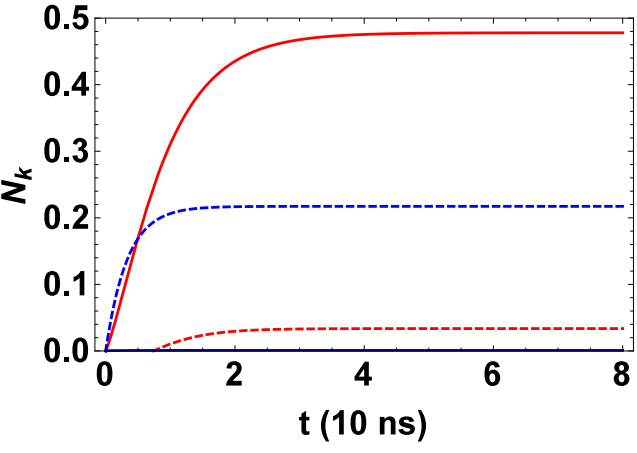

(b)

FIG. 3: (a) $N_{k}$ ( $\mathrm{k}=\mathrm{SX}$ (red solid line), TX (red dashed line), SCT (blue solid line), TCT (blue dashed line)) as a function of time, $t$, obtained by numerically solving Eqs. [ to 4 using Eq. 8 , and setting $N_{i}=1, \tau=10 \mathrm{~ns}, \zeta=0$. We also choose $\beta=\frac{\tau_{S C T}}{\tau_{T C T}}=0.001, \gamma=\frac{\tau_{I S C}}{\tau_{T C T}}=0.5, \frac{\tau_{S X}}{\tau_{T C T}}=0.7, \frac{\tau_{T X}}{\tau_{T C T}}=0.5$ with $\tau_{T C T}=10 \mathrm{~ns}$. (b) Same values for all parameters as in (a), with exception of $\zeta=0.15$ (see Eq. 8), and $\alpha_{s}=\frac{\tau_{S X}}{\tau_{S p}}=1, \alpha_{p}=\frac{\tau_{T X}}{\tau_{T p}}=1$

\section{CONCLUSION}

In conclusion, we have derived an analytical relation of the singlet exciton yield that incorporates the Pauli exclusion mechanism via the purity measure, $P$. Our model considers the formation of singlet excitons via inter-conversion from loosely bound charge-transfer (CT) triplet electron-hole pair. We include a channel by which CT triplet states are formed from tightly bound excitons at high densities. Hence we have taken into account the crucial mechanism by which singlet excitons are increased by preventing the loss of triplet exciton which otherwise would occur through recombination and other dissipation processes. Non-ideal triplet excitons are rerouted via the Pauli exclusion mechanism to form loosely bound CT states which subsequently convert to singlet excitons. As a consequence, we obtain the fluorescence quantum yield that can increase beyond the well known estimate of $>25 \%$. This is the main result of this work which has importance in conjugated polymers systems. The theoretical framework presented in this work provides a possible explanation for the high exciton yields noted in earlier works $\$ 3.19,20$ and will be useful in evaluating optimal electroluminescence efficiencies in organic devices.

The idea of entanglement control by structural variations in novel quantum dot nanostructures ${ }^{42,43}$ may be employed to seek enhancement of the singlet exciton yield in polymer systems. The incorporation of the Pauli exclusion principle during diffusion of photogenerated excitons in drift-diffusion models $\underline{44}$ will also assist in the prediction of realistic 


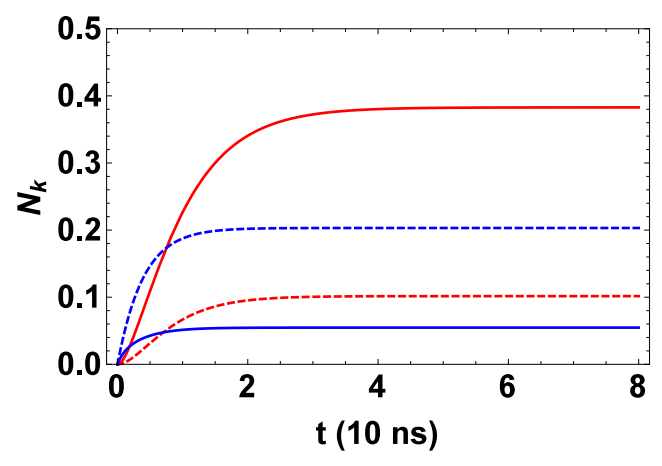

(a)

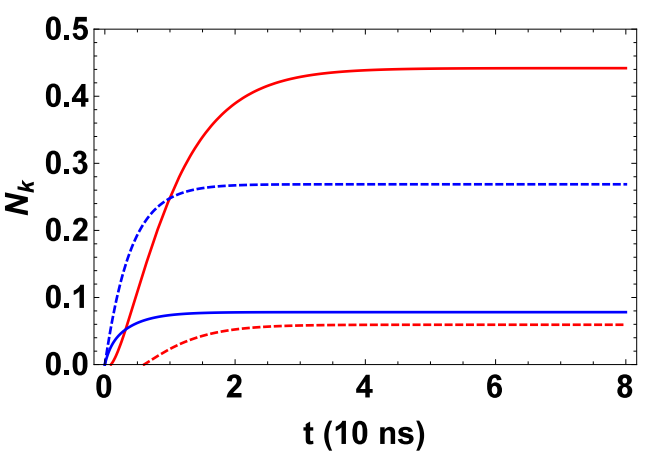

(b)

FIG. 4: (a) $N_{k}$ ( $\mathrm{k}=\mathrm{SX}$ (red solid line), TX (red dashed line), SCT (blue solid line), TCT (blue dashed line)) as a function of time, $t$, obtained by numerically solving Eqs. 1 to 4 using Eq. 8 , and setting $N_{i}=1, \tau=10$ ns, $\zeta=0$. We set $\beta=\frac{\tau_{S C T}}{\tau_{T C T}}=0.1, \gamma=\frac{\tau_{I S C}}{\tau_{T C T}}=0.5, \frac{\tau_{S X}}{\tau_{T C T}}=0.7, \frac{\tau_{T X}}{\tau_{T C T}}=0.5$ with $\tau_{T C T}=10 \mathrm{~ns}$. (b) Same values for all parameters as in (a), with exception of $\zeta=0.15$, and $\alpha_{s}=\frac{\tau_{S X}}{\tau_{S p}}=1, \alpha_{p}=\frac{\tau_{T X}}{\tau_{T p}}=1$

operating conditions in polymer based devices with morphologies of different dimensions $\underline{45}-\underline{47}$. To this end, future experimental investigations may consider the control of the entanglement attributes of excitons through polymer length tailoring and engineering of morphological attributes 48 . The incorporation of the Pauli exclusion principle in determining the efficiency of devices composed of other material systems such as CdSe and PbSe nanocrystals $\underline{49}$ where multiple excitons are generated, and layered transition metal dichalcogenides with enhanced excitonic properties $\underline{50} \underline{54}$ may be explored in future works. Future experimental works should seek to investigate alternative means of targeting the triplet excitons to increase the singlet exciton yield.

\section{ACKNOWLEDGMENTS}

This research was undertaken on the NCI National Facility in Canberra, Australia, which is supported by the Australian Commonwealth Government.

* Electronic address: thilaphys@gmail.com

1 M. Baldo, S. Forrest, Physical Review B 62(16), 10958 (2000)

2 J. Wilson, A. Dhoot, A. Seeley, M. Khan, A. Köhler, R. Friend, Nature 413(6858), 828 (2001)

3 M. Wohlgenannt, K. Tandon, S. Mazumdar, S. Ramasesha, Z. Vardeny, Nature 409(6819), 494 (2001)

${ }^{4}$ R. Kersting, U. Lemmer, M. Deussen, H. Bakker, R. Mahrt, H. Kurz, V.I. Arkhipov, H. Bässler, E. Göbel, Physical Review letters 73(10), 1440 (1994)

5 T.Q. Nguyen, I.B. Martini, J. Liu, B.J. Schwartz, The Journal of Physical Chemistry B 104(2), 237 (2000)

${ }^{6}$ R. Friend, R. Gymer, A. Holmes, J. Burroughes, R. Marks, C. Taliani, D. Bradley, D. Dos Santos, J. Bredas, M. Lögdlund, et al., Nature 397(6715), 121 (1999)

7 J. Burroughes, D. Bradley, A. Brown, R. Marks, K. Mackay, R. Friend, P. Burns, A. Holmes, nature 347(6293), 539 (1990)

${ }^{8}$ W. Barford, Physical Review B 70(20), 205204 (2004)

9 T.A. Skotheim, J. Reynolds, Conjugated polymers: theory, synthesis, properties, and characterization (CRC press, 2006)

10 S.A. Jenekhe, et al., Excimers and exciplexes of conjugated polymers. Tech. rep., DTIC Document (1994)

11 Z. Shuai, D. Beljonne, R. Silbey, J.L. Brédas, Physical Review letters 84(1), 131 (2000)

12 S. Günes, H. Neugebauer, N.S. Sariciftci, Chemical Reviews 107(4), 1324 (2007)

13 H. Sirringhaus, P. Brown, R. Friend, M.M. Nielsen, K. Bechgaard, B. Langeveld-Voss, A. Spiering, R.A. Janssen, E. Meijer, P. Herwig, et al., Nature 401(6754), 685 (1999)

14 X. Liu, Y. Sun, B.B. Hsu, A. Lorbach, L. Qi, A.J. Heeger, G.C. Bazan, Journal of the American Chemical Society 136(15), $5697(2014)$

15 M. Wohlgenannt, K. Tandon, S. Mazumdar, S. Ramasesha, Z. Vardeny, Nature 409(6819), 494 (2001)

16 L. Yao, B. Yang, Y. Ma, Science China Chemistry pp. 1-11 (2014)

17 Z. Shuai, Q. Peng, Physics Reports (2013) 
18 M. Carvelli, R. Janssen, R. Coehoorn, Physical Review B 83(7), 075203 (2011)

19 J.S. Kim, P.K. Ho, N.C. Greenham, R.H. Friend, Journal of Applied Physics 88(2), 1073 (2000)

20 L. Lin, H. Meng, J. Shy, S. Horng, L. Yu, C. Chen, H. Liaw, C. Huang, K. Peng, S. Chen, Physical Review letters 90(3), 036601 (2003)

21 B.J. Schwartz, Annual review of physical chemistry 54(1), 141 (2003)

22 S. Karabunarliev, E.R. Bittner, Physical Review letters 90(5), 057402 (2003)

23 C. Law, Phys. Rev. A 71, 034306 (2005).

24 M. Combescot, EPL (Europhysics Letters) 96(6), 60002 (2011)

25 M. Combescot, O. Betbeder-Matibet, F. Dubin, Physics Reports 463(5), 215 (2008)

26 M. Combescot, F. Dubin, M. Dupertuis, Physical Review A 80(1), 013612 (2009)

27 C. Chudzicki, O. Oke, W.K. Wootters, Physical Review letters 104(7), 070402 (2010)

28 A. Gavrilik, Y.A. Mishchenko, Physics Letters A 376(19), 1596 (2012)

29 P. Kurzyński, R. Ramanathan, A. Soeda, T.K. Chuan, D. Kaszlikowski, New Journal of Physics 14(9), 093047 (2012)

30 M.C. Tichy, P.A. Bouvrie, K. Mølmer, Physical Review letters 109(26), 260403 (2012)

31 M.C. Tichy, P.A. Bouvrie, K. Mølmer, Physical Review A 86(4), 042317 (2012)

32 A. Thilagam, arXiv preprint arXiv:1407.1091 (2014)

33 S.M. Hashemi Rafsanjani, in Frontiers in Optics (Optical Society of America, 2010)

34 A. Thilagam, Journal of Mathematical Chemistry 51(7), 1897 (2013)

35 A. Thilagam, Physical Review B 63(4), 045321 (2001)

36 A. Köhler, D. Beljonne, Advanced Functional Materials 14(1), 11 (2004)

37 P. Blom, M. De Jong, J. Vleggaar, Applied Physics Letters 68(23), 3308 (1996)

38 A. Thilagam, Physical Review A 81(3), 032309 (2010)

39 R. Friend, R. Gymer, A. Holmes, J. Burroughes, R. Marks, C. Taliani, D. Bradley, D. Dos Santos, J. Bredas, M. Lögdlund, et al., Nature 397(6715), 121 (1999)

40 J. W. Yu, J. K. Kim, D. Y. Kim, C. Kim, N. W. Song, D. Kim, Curr. Appl. Phys., 6, 59 (2006).

41 M.C. Tichy, P.A. Bouvrie, K. Mølmer, Applied Physics B 117 (3), 785 (2014).

42 J. Coe, S. Abdullah, I. DAmico, Journal of Applied Physics 107(9), 09E110 (2010)

43 S. Abdullah, J. Coe, I. DAmico, Physical Review B 80(23), 235302 (2009)

44 G.A. Buxton, N. Clarke, Modelling and simulation in Materials Science and Engineering 15(2), 13 (2007)

45 H.K. Kodali, B. Ganapathysubramanian, Modelling and Simulation in Materials Science and Engineering 20(3), 035015 (2012)

46 M. Hoffmann, K. Schmidt, T. Fritz, T. Hasche, V. Agranovich, K. Leo, Chemical Physics 258(1), 73 (2000)

47 Y. Min Nam, J. Huh, W. Ho Jo, Solar Energy Materials and Solar Cells 94(6), 1118 (2010)

48 W. Ma, C. Yang, X. Gong, K. Lee, A.J. Heeger, Advanced Functional Materials 15(10), 1617 (2005)

49 R.D. Schaller, V.M. Agranovich, V.I. Klimov, Nature physics 1(3), 189 (2005)

${ }^{50}$ K.F. Mak, K. He, C. Lee, G.H. Lee, J. Hone, T.F. Heinz, J. Shan, Nature materials 12(3), 207 (2013)

51 A. Ramasubramaniam, Physical Review B 86(11), 115409 (2012)

52 J. Wilson, A. Yoffe, Advances in Physics 18(73), 193 (1969)

53 Q.H. Wang, K. Kalantar-Zadeh, A. Kis, J.N. Coleman, M.S. Strano, Nature Nanotechnology 7(11), 699 (2012)

54 A. Thilagam, Journal of Applied Physics 116(5), 053523 (2014) 